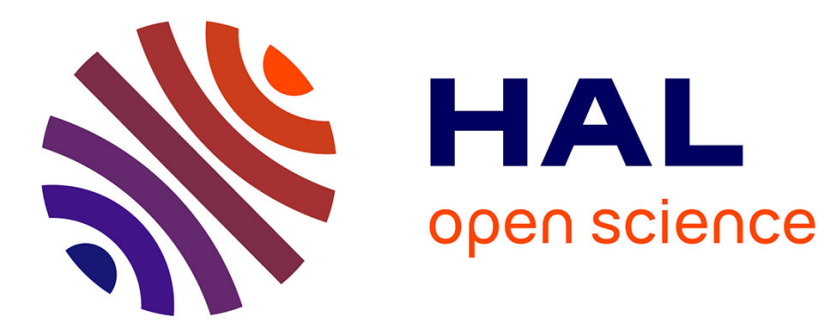

\title{
La nuit, nouveau territoire des politiques publiques
}

\author{
Luc Gwiazdzinski
}

\section{To cite this version:}

Luc Gwiazdzinski. La nuit, nouveau territoire des politiques publiques. L'Observatoire, la revue des politiques culturelles , 2019, 53, pp.11-15. 10.3917/lobs.053.0011 . halshs-01985584

\section{HAL Id: halshs-01985584 https://shs.hal.science/halshs-01985584}

Submitted on 18 Jan 2019

HAL is a multi-disciplinary open access archive for the deposit and dissemination of scientific research documents, whether they are published or not. The documents may come from teaching and research institutions in France or abroad, or from public or private research centers.
L'archive ouverte pluridisciplinaire HAL, est destinée au dépôt et à la diffusion de documents scientifiques de niveau recherche, publiés ou non, émanant des établissements d'enseignement et de recherche français ou étrangers, des laboratoires publics ou privés. 


\author{
La nuit nouveau territoire des politiques publiques \\ Revue L’Observatoire des politiques publiques, n53, hiver 2019, pp.7-9
}

\title{
Luc Gwiazdzinski (*)
}

\author{
"Quels mortels, quel être doué de la faculté de sentir, \\ ne préfère pas au jour fatigant la douce lumière de la nuit \\ avec ses couleurs, ses rayons, ses vagues flottants qui se répandent partout »
}

Novalis

Espace-temps longtemps peu investi, la nuit est devenue un territoire sous pression, à la fois reflet et moteur des mutations des sociétés contemporaines. Muse et refuge traditionnel des artistes, la nuit est à la fois un observatoire, un marché investi par les acteurs économiques et le marketing, un support de revendications (droit à la ville, genre, jeunesse, nature, etc.), un espace-temps de mobilisation pour les citoyens, un territoire d'expérimentation et un nouvel enjeu pour les politiques publiques. Caricature du jour, elle révèle l'homme et l'humanité dans ses potentiels et ses contradictions. Mieux, elle nous apprend beaucoup sur le jour et sur nos futurs possibles.

Selon la Genèse, "Dieu sépara la lumière des ténèbres. Il appela la lumière "jour” et les ténèbres "nuit". Il y eut un soir, il y eut un matin : premier jour ». L'homme et la ville ont toujours eu une existence rythmée par l'alternance jour-nuit. Mais les temps changent. Audelà des rêves, des peurs et des fantasmes, il y a désormais une vie après le jour. La nuit n'est plus la période d'obscurité complète symbolisée par le couvre-feu, la fermeture des portes de la cité et le repos social qui inspirait les artistes en quête de liberté, servait de refuge aux malfaiteurs et inquiétait les pouvoirs en place qui cherchaient à la contrôler. Économie, environnement, social et culture, la marge oubliée d'hier devient un espace-temps central pour les entreprises, les collectivités, les territoires et les citoyens.

\section{Une marge investie par les artistes}

Il y a quelques années encore, la nuit urbaine, intéressait peu les chercheurs, les édiles et les techniciens de l'aménagement et de l'urbanisme qui pensaient et géraient la ville comme une entité fonctionnant seulement $16 \mathrm{~h} / 24$ et 5 jours/7. Dans nos agendas papier, la journée s'arrêtait généralement à $21 \mathrm{~h}$ et il était impossible d'y indiquer un rendez-vous de nuit. Dans de nombreuses langues, le mot «nuit » apparaît même comme une négation des huit heures : non-huit ; no-eight : nein-acht ; non-otto ; no-ocho. Puissant stimulant de l'imagination, la nuit a souvent inspiré les artistes qui s'y sont réfugiés fuyant les normes et les injonctions du grand jour pour créer et résister. Les musiciens - puis les peintres - en ont fait un genre à part, les Nocturnes dans laquelle ont excellé Mozart et Chopin. En littérature et en poésie, des chantres aussi talentueux que Novalis, Michaux, Apollinaire, Saint-John Perse, Char, Breton ou Aragon, se sont laissés porter par la nuit urbaine. Le roman noir est devenu un genre à part qui joue magnifiquement avec la nuit. Comme les poètes, les photographes, écrivains de la lumière, ont souvent su aller dénicher ce qui reste de jour au fond de la nuit. Paris la nuit de Brassaï révéla en noir et blanc toute la poésie des lampadaires et des pavés mouillés imposant une certaine esthétique nocturne. Le cinéma s'est emparé de la nuit pour la réinventer et transformer notre approche, jouant habilement sur les ambiguïtés et les contradictions en 
profitant de la magie des salles obscures. Le septième art a fait de la nuit un véritable personnage avec quelques chefs d'œuvre entre Paris qui dort (1923) de René Clair et Les Nuits fauves de Cyril Collard (1992). L'expression artistique est sans doute plus adaptée que les sciences pour décliner les variations de la nuit, mettre en évidence les tensions, jouer des équilibres entre les pleins de la raison et les vides du songe, mais aussi pour jongler avec les paradoxes.

\section{Une conquête progressive par la lumière et le pouvoir}

Pendant des millénaires, l'Homme n'a eu de cesse de chercher à contrôler l'autre partie du jour. La généralisation de l'éclairage public et l'affirmation du pouvoir politique ont permis la conquête de la nuit. En quelques siècles, on est passé de la « ville de garde » (sécurité, santé), où l'activité demeurait une exception surveillée, aux loisirs d'une élite. La démocratisation de la nuit festive et l'apparition du by night arrivent avec la fin du XIX ${ }^{\mathrm{e}}$ siècle. Cette longue conquête s'est accélérée au cours des trente dernières années. La nuit se banalise et même les lois évoluent: autorisation du travail de nuit des femmes, chasse nocturne, perquisitions. C'est désormais toute l'économie du jour qui s'intéresse à la nuit contribuant à sa «diurnisation», phase ultime de «l'artificialisation» de la ville. D'autres populations, d'autres points de convergence, d'autres limites et d'autres frontières se dessinent dans la nuit qui devient un territoire d'investigation, de créativité et d'expérimentation.

\section{Une évolution des rythmes et des pratiques nocturnes}

La ville revoit ses nycthémères et même les rythmes biologiques semblent bouleversés. On s'endort en moyenne à $23 \mathrm{~h}$ au lieu de $21 \mathrm{~h}$, il y a cinquante ans et l'on dort de moins en moins. La lumière a progressivement pris possession de l'espace urbain, gommant en partie l'obscurité menaçante de nos nuits, permettant la poursuite d'activités diurnes et l'espace nocturne s'est transformé en un terrain d'expérimentation. Le couvre-feu médiatique est désormais terminé : radios et télévisions fonctionnent $24 \mathrm{~h} / 24$ et Internet permet de surfer avec des régions du monde où il fait jour. Toutes les foires et salons ont désormais leurs «nocturnes» et les «soldes de nuit» attirent les foules. Les entreprises industrielles produisent souvent en continu pour rentabiliser leurs équipements et, dans la plupart des secteurs, le travail de nuit se banalise atteignant près de $20 \%$ des salariés. Les commerces décalent leurs services vers le soir. Il y a des années déjà que les sociétés de services sont au " 24h/24, 7j/7 ». Progressivement les activités humaines se sont déployées dans la nuit et ont recomposé un nouvel espace-temps de travail, de loisirs et de culture. Les pratiques changent et l'espace public mute. $80 \%$ des Français déclarent sortir en ville la nuit contre moins de vingt pour cent il y a trente ans. Le téléphone portable a révolutionné la pratique des nuits urbaines dans une logique de «temps réel». Les usagers de la ville la nuit " consomment» désormais la ville sous forme de parcours nocturnes nomadisant d'un pôle d'attraction à l'autre et compliquant le contrôle des nuisances sonores. La législation sur la consommation de tabac dans les espaces publics a également renvoyé une partie des consommateurs dans la rue permettant de nouvelles formes de socialisation, une reconquête de l'espace public mais multipliant également les tensions et conflits avec les résidants. Individus, groupes et quartiers de la ville polychronique qui ne vivent plus aux mêmes rythmes ne font plus toujours bon ménage. Ils obligent à investir la nuit pour imaginer des solutions. 


\section{Une actualité diurne}

Reflet et conséquence de ces mutations, la nuit s'est imposée dans l'actualité du jour pour le meilleur (fêtes, événements...) et pour le pire (violences urbaines, conflits, nuisances sonores, insécurité, pollution lumineuse...). La dernière frontière ${ }^{1} \mathrm{~d}^{\prime}$ hier est devenue un espace-temps central, un sujet de société qui s'étale des pages des revues scientifiques ${ }^{2}$ à celles de la presse nationale et régionale en passant par les revues de vulgarisation ${ }^{3}$ ou de marketing ${ }^{4}$. Depuis quelques années, les publications scientifiques, les programmes de recherche et les plateformes partenariales se structurent - comme la Plateforme nationale de la vie nocturne ${ }^{5}$. Un nouveau champ de recherches interdisciplinaire, celui des Night studies émerge peu à peu. Les colloques ${ }^{6}$, séminaires, travaux de recherche, thèses et expositions sur la nuit (Nuits parisiennes $^{7}$, Peindre la nuit $^{8}$ ) se multiplient.

\section{Des mobilisations et une mise à l'agenda politique}

À côté des collectifs de résidents traditionnellement opposés au développement de la nuit festive, d'autres acteurs se fédèrent pour défendre leur nuit. Parfois des milliers de personnes se mobilisent comme à Tbilissi (Georgie) en mai 2018 pour manifester contre des opérations de police dans des boîtes de nuit transformant la place du parlement en discothèque géante avec un message clair : vous prenez le contrôle de nos clubs, nous prenons le contrôle de la ville. En France, lors des élections municipales de 2014, les mobilisations citoyennes ont notamment pris la forme d'élections de " maires de la nuit », à l'imitation d'Amsterdam aux Pays-Bas où, depuis 2003, les acteurs de la nuit ont élu leur Nachtbrugermeester. On en compte désormais une quarantaine dans le monde - aux compétences et au mode de désignation divers - de Toulouse à Nantes en passant par Vilnius, ou Mannheim où le maire de la nuit a été nommé en juillet 2018, par un jury composé de représentants de la municipalité, de citoyens et de professionnels de la nuit. Avec Nuit debout en 2016, la dimension politique de la nuit a également ressurgi sur les places des villes de France en écho au «mouvement des places». La nuit devient un support pour des revendications de développement durable à l'instar de l'opération le Jour de la Nuit sur la protection de la biodiversité nocturne et du ciel étoilé et contre la pollution lumineuse. Des opérations de sensibilisation invitent à prendre soin de la nuit comme le Manifesto da Noite à Sao Paulo. Signe des temps, les loisirs nocturnes sont à la mode à l'exemple de L'atelier des lumières à Paris, un centre d'art numérique où le public est immergé dans les projections de tableaux de maîtres.

\section{Vers des politiques publiques de la nuit}

Ces tensions, les changements de modes de vie, la demande de services adaptés, la mise en compétition des villes, ont obligé les pouvoirs publics à réagir, pour développer d'autres approches en termes de régulation, de médiation ou d'animation transformant la nuit en

\footnotetext{
${ }^{1}$ L. Gwiazdzinski, La nuit dernière frontière de la ville, La Tour d'Aigues, Éditions de l'Aube, 2005 (ré-édition 2016, Rhuthmos).

${ }^{2}$ Hémisphères, La revue suisse de la recherche et de ses applications, HES SO(Haute école spécialisée de Suisse occidentale), volume XII, 2018.

${ }^{3}$ Socialter $\mathrm{n}^{\circ} 29$, juin-juillet 2018

${ }^{4}$ La revue des marques $\mathrm{n}^{\circ} 97$, janvier 2017.

${ }^{5} \mathrm{http} / / /$ pnvn.org/a-propos/

${ }^{6} 3$ èmes rencontres européennes de la vie nocturne,1, 2, 3 février 2018 à Lyon / NIGHTS 2018 - 4ème Conférence internationale sur la vie nocturne 15-17 novembre - Bruxelles

${ }^{7}$ Du 25 novembre 2017 au 27 janvier 2018, 1'exposition Les Nuits parisiennes a retracé deux siècles et demi de vie nocturne dans la capitale et a rassemblé plus de 300 tableaux, photos, décors, films.

${ }^{8}$ Du 13 octobre 2018 au 15 avril 2019, le Centre Pompidou-Metz consacre une exposition de grande ampleur au thème de la nuit dans la peinture moderne et contemporaine.

${ }^{9}$ Night Manifesto. Seeking Citizenship 24h, São Paulo, Invisiveis Produçoes, 2014.
} 
territoire d'expérimentation. Depuis une quinzaine d'années, on assiste à la «mise à l'agenda » progressive de la nuit urbaine. Face aux pressions, les autorités cherchent à la fois à conserver le contrôle (règlementation des événements, couvre-feux, arrêtés municipaux, etc.) et à développer des activités (éclairages, événements festifs gratuits, transports, crèches, etc.) pour rendre les nuits urbaines plus accessibles et hospitalières, participant de la sorte à l'accroissement de la flexibilité. En Europe, les initiatives se multiplient dans trois directions principales : l'amélioration de la qualité de vie des habitants à travers de nouveaux services ou l'extension d'activités diurnes, l'animation nocturne dans une logique de marketing territorial et d'attractivité - dans laquelle la lumière joue un rôle important - et la tranquillité publique ou sécurité. La dynamique est là, même si on ne peut pas encore véritablement parler de «politiques publiques de la nuit» au sens «d'un ensemble d'actions coordonnées, mises en cuvre avec pour objectif d'obtenir une modification ou une évolution d'une situation donnée ${ }^{10}$.

\section{De l'exploration à la médiation}

De nombreuses collectivités cherchent d'abord à mieux connaître la nuit en mettant à plat les connaissances et en partageant les diagnostics. Après les premiers travaux sur l' « économie de la nuit » des années 90 à Leeds ou Manchester, d'autres villes ont cherché à mieux connaître leurs nuits comme Londres, Gloucester, Leicester mais également Sydney, Melbourne, Montréal ou Big apple. Des États généraux de la nuit ont été organisés dans de nombreuses villes comme Paris, Genève ou Lausanne à l'initiative des collectivités souvent poussées par des associations et certaines villes (Bruxelles, Lyon, etc.) ont créé des observatoires de la nuit. Depuis une vingtaine d'années, des dizaines de «traversées nocturnes » ont permis de construire des diagnostics sensibles et partagés (Genève ${ }^{11}$, Milan, Montréal, Porto, Strasbourg, Grenoble, etc.) et de contribuer à la sécurisation des parcours nocturnes des femmes. En 2018, Paris a organisé une Nuit de la solidarité pour dénombrer les sans-abris et adapter ses dispositifs ${ }^{12}$ à laquelle ont participé près de 2000 personnes.

La médiation est un autre élément important des politiques publiques qui se déploient dans la nuit. L'expérience des Correspondants de nuit de Rennes ou de Strasbourg a essaimé dans de nombreuses villes. À Barcelone et à Montréal, des " chuchoteurs » se sont installés près des établissements de nuit afin de réduire les nuisances. À Paris, le dispositif des Pierrots de la nuit mobilise des artistes. Des démarches de médiation se déploient, comme les Chartes de la nuit ou de la vie nocturne (Lille, Rennes, Lyon, Strasbourg, Besançon etc.) pour améliorer le dialogue entre résidents, établissements et consommateurs. Face à l'explosion du nombre de SDF, les plans d'accueil d'urgence et les maraudes se développent dans toutes les villes. Dans toute la France en décembre, 141244 places sont désormais ouvertes, soit une augmentation de $10 \%{ }^{13}$.

\section{Extension du domaine du jour}

Dans tous les pays du monde, la tendance générale est à une augmentation de la périodicité, de l'amplitude et de la fréquence des transports publics en soirée et la nuit. En région parisienne, le réseau Noctilien remporte un large succès. À Londres, le métro est désormais

\footnotetext{
${ }^{10}$ J.-C. Thoenig, «Politique publique », in L. Boussaguet et al., Dictionnaire des politiques publiques, Éditions de Sciences Po, 2010, pp. $420-427$.

${ }^{11}$ Vidéo « Genève explore sa nuit », https://www.youtube.com/embed/azkGCohcmGE

12 «Nuit de la solidarité organisée par la mairie de Paris pour dénombrer les sans-abri », in Le Monde,15 février 2018.

${ }^{13}$ Dispositif hivernal 2017-2018. L'accueil, l'hébergement et l'accompagnement des personnes sans-abri, 29 décembre 2017, Ministère de la cohésion sociale.
} 
ouvert toute la nuit. Depuis des années, Helsinki propose des crèches de nuit pour faciliter la vie des salariés de nuit, imitée par d'autres villes. Dans les Asturies, l'ouverture des centres sociaux et des gymnases en début de nuit a permis de faire baisser la délinquance juvénile et Rennes a suivi avec sa Nuit des quatre jeudis. De nombreuses bibliothèques et universités sont ouvertes la nuit aux États-Unis et dans quelques villes européennes. En matière de politiques culturelles et sportives, de nombreux équipements (piscines, gymnases, centre culturels...) ouvrent plus tard en soirée. À Paris, les réflexions ${ }^{14}$ ont abouti à de nombreuses expérimentations : une quinzaine de parcs et jardins accessibles la nuit, une boite de nuit sur une péniche ouverte $24 \mathrm{~h} / 24$, trois centres d'animation ouverts jusqu'à $2 \mathrm{~h}$ du matin, une vingtaine de piscines accessibles de $20 \mathrm{~h}$ à $23 \mathrm{~h}$ au moins un soir par semaine, une soixantaine d'équipements sportifs ouverts entre $22 \mathrm{~h} 30$ et minuit et une expérimentation du STIF (1'arrêt à la demande dans les bus de nuit) afin de lutter contre le harcèlement des femmes.

Les politiques d'éclairage public se poursuivent avec notamment la mise en place de schémas lumière et la modernisation de l'éclairage public dans une logique de confort des habitants et de transition énergétique parfois sous forme de régie comme à Grenoble. La fonction de l'éclairage public change progressivement, passant de la sécurité à l'agrément. Les illuminations de bâtiments se multiplient et des artistes comme Yann Kersalé sculptent la nuit offrant une identité nocturne à nos cités. Parallèlement, les sons et lumières se déclinent désormais à toutes les échelles loin de la première expérience de Chambord dans les années cinquante. La Fête des lumières de Lyon n'est qu'une manifestation parmi une soixantaine recensées. Entre découverte artistique et nouveau tourisme urbain, le calendrier nocturne s'épaissit à l'initiative des pouvoirs publics ou d'acteurs privés : Nuit des arts, Nuit des musées, Nuits blanches de Saint-Pétersbourg, Paris, Rome, Bruxelles, Montréal ou Naples, Nuit européenne de la science à Berlin et ailleurs.

Les qualités supposées de la nuit comme espace d'échanges et de créativité prennent la forme de manifestations aux noms évocateurs (Nuit des débats, des idées, de la philosophie, de la géographie, du volley, des infirmières...). En milieu rural les «marchés nocturnes » sont désormais intégrés dans des dynamiques de développement local. alors que des « réserves d'obscurité » et des labels tels que « villes et villages étoilés » ${ }^{15}$ sont créés contre la pollution lumineuse et pour « la biodiversité nocturne ».

Enfin, dans de nombreuses villes comme Nantes, Strasbourg ou Paris, des «élus désignés » sont désormais en charge de la question transversale de la nuit répondant enfin à l'initiative citoyenne des «Maires de nuit» mais sans reprendre l'idée d'élections de représentants du peuple de la nuit ${ }^{16}$. Ailleurs, les acteurs ont préféré mettre en place des structures originales comme le Grand conseil de la nuit à Genève. Dans ce cadre, des appels à projets et des chantiers sont lancés avec la population. À Nantes, qui développe depuis trois ans une vraie politique de la vie nocturne, le Conseil de la nuit a annoncé la refonte du service de bus de nuit, une offre de "stations nocturnes » lieux dits «de transition » sécurisants pour les noctambules, mais aussi des "ateliers sur le genre », des mariages de nuit (19h-23h), des points d'eau pour les SDF et les prostituées, l'expérimentation de « spots à pipi nocturnes » et l'ouverture nocturne des toilettes Decaux pour les femmes.

\footnotetext{
${ }^{14}$ B. Badia, D. Bertrand, A. Carrera, L. Gwiazdzinski, P. Kertudo, «L'évolution des usages publics nocturnes à Paris », in revue Recherche sociale $\mathrm{n}^{\circ} 206$, avril-juin 2013, pp.6-75.

${ }^{15}$ Créé par l'Association Nationale pour la Protection du Ciel et de l'Environnement Nocturnes (ANPCEN).

${ }^{16}$ Gwiazdzinski L., 1999, « Entre ombre et lumière », Revue Aménagement et nature n ${ }^{\circ}$ 33, juin 1999, p.105-108
} 


\section{Les possibles apports d'une « pensée nuitale »}

La nuit, en tant que territoire d'expérimentation, est également une singulière expérience. Elle nous enveloppe et nous pénètre par tous les sens. Loin des certitudes et des blocages de la société diurne, la nuit met en évidence toute l'importance de «l'habiter », à la fois mode de connaissance du monde et «type de relations affectives loin d'une approche abstraite ou technocratique de l'espace ». La nuit est l'occasion de passer d'une pensée de l'organiser à une pensée de 《l'exister $»{ }^{17}$. Elle permet d'échapper aux valeurs du jour, celles de la raison et de la modernité toujours chargées positivement pour introduire de l'humain, du sensible, de l'entre-deux, loin des réponses binaires. Une " pensée nuitale » peut permettre de gérer les contradictions et paradoxes d'une société et d'individus "hypermodernes » : éclairer la nuit sans pour autant la tuer ; rendre la nuit accessible et préserver son identité originelle ; développer la nuit sans créer de nouveaux conflits d'usage ; animer la nuit et respecter nos rythmes biologiques ; assurer la sécurité publique sans imposer un couvre-feu ; ouvrir la nuit tout en préservant la santé des travailleurs ; assurer la continuité centre-périphérie sans uniformiser la nuit; réguler la nuit tout en conservant une place pour la transgression; ne pas tout réglementer sans pour autant abandonner la nuit au marché ; développer l'offre de services et conserver le silence et l'obscurité ; concilier "droit à la ville » et «droit à la nuit ${ }^{18}$ et enfin investir la nuit tout en lui conservant une part de mystère. " L'urbanisme de la nuit » pourrait s'en inspirer et s'appuyer sur les notions d'hospitalité, de qualité, d'égalité, de sensibilité, de variété, d'inattendu et de sécurité par l'accroissement de la présence humaine et l'enchantement. Poussés vers le jour, ces principes de «ménagement de la nuit» pourraient contribuer à un « urbanisme des liens, des interactions et des médiations » plutôt qu'à un urbanisme de séparation et de zonage. Partager plutôt que séparer. Contractualiser plutôt qu'imposer.

\section{Nocturnes ménagements}

Économie, culture, social et environnement: les politiques publiques de la nuit restent à construire ensemble en instaurant quelques règles et garde-fous. Les risques d'appropriation, de confiscation voire de privatisation du silence, de l'obscurité, de la vacance et du vide obligent à aborder la nuit comme patrimoine et «bien commun ». Faisons en sorte que les nuits conservent leur différence et qu'elles ne deviennent pas l'équivalent du jour. Veillons à ce qu'elles demeurent des espaces de liberté et de création laissant la place à l'inorganisé, à l'improvisation, au mystère et à la distraction loin de l'hyper-connectivité de la smart city. Évitons la transparence, la fin de l'ombre et la lumière carcérale et suivons les mises en garde des chronobiologistes: "sans rythme pas de vie ${ }^{19}$. Loin des certitudes et des blocages de la société diurne, on peut encore rêver d'une ville plus habitable, humaine, accessible et hospitalière de nuit comme de jour. À ce sujet, les propositions des lettristes nous interpellent encore : "Munir les réverbères de toutes les rues d'interrupteurs ; l'éclairage étant à la disposition du public $»^{20}$. Pour celles et ceux qui veulent bien prendre soin de la nuit, la question centrale est sans doute l'apprentissage de la mesure : sans lumière pas de ville la nuit, mais trop de lumière tue la nuit. Prendre soin de la nuit, c'est prendre soin de nous.

\footnotetext{
${ }^{17}$ H. Maldiney H., 2007, « La rencontre et le lieu », in C. Younes C. (Dir.), Henry Maldiney. Philosophie, art et existence. La nuit surveillée, Cerf, 2007, $222 \mathrm{p}$.

${ }^{18}$ Night Manifesto. Seeking Citizenship 24h, 2014, São Paulo, Invisiveis Produçoes.

${ }^{19}$ B. Millet, «L'homme dans la ville en continu », in L. Gwiazdzinski, La ville 24h/24, La Tour d'Aigues, L'Aube, 2003 (2 ${ }^{\text {nde }}$ éd., 2016 Rhuthmos), pp.83-91.

${ }^{20}$ Potlach n ${ }^{\circ} 23,1955$.
} 


\section{Indications bibliographiques :}

Schivelbusch W., 1993, La Nuit désenchantée, Le Promeneur, Paris

Bohringer, R. 2006. C'est beau une ville la nuit, Paris, Denoël.

Cabantous A., 2009, Histoire de la nuit: XVIIe-XVIIIe siècle, Paris, Fayard.

Castellini A., 2003, Piacevole è la notte. Milan, Manifestolibri.

Chatterton P., Robert H., 2003, Urban Nightscapes: Youth Cultures, Pleasure Spaces and Corporate Power, New York, Routledge.

Crary J. 2007. 24/7. Late Capitalism and the Ends of Sleep, London, New-York, Verso.

Deleuil, J.-M. (dir.), 2009, Éclairer la ville autrement. Innovations et expérimentations en éclairage public, Lausanne, Presses universitaires et polytechniques romandes.

Durand G., 1960. Les structures anthropologiques de l'imaginaire, Paris, Dunod.

Delattre, S. 2004. Les douze heures noires. La nuit à Paris au XIXème siècle, Paris, Albin Michel.

Espinasse C., Heurgon E., Gwiazdzinski L. (Dir.), 2005, La nuit en question(s), Editions de l'Aube (ré-édition 2017, Hermann)

Gwiazdzinski L., 2016, " Nuit debout, Première approche du régime de visualité d'une scène nocturne », Revue Imaginations, Visualités http://imaginations.csj.ualberta.ca/

Gwiazdzinski, L. 2007. Nuits d'Europe. Pour des villes accessibles et hospitalières, Belfort, UTBM Editions.

Gwiazdzinski, L. 2005. La nuit dernière frontière de la ville, La Tour d'Aigues, Éditions de l'Aube (ré-édition 2016, Rhuthmos)

Gwiazdzinski, L. 2003. La ville 24h/24, La Tour d'Aigues, Éditions de l'Aube (ré-édition 2016, Rhuthmos)

Gwiazdzinski, L. 2002. " Aménager la nuit urbaine », Le Monde, 6 octobre 2002.

Kersale, Y. 2008. Manière noire. Géopoétique du paysage, Paris, Éditions L'une et l'autre.

Koslovsky C. 2011. Evening's Empire, Cambridge University Press.

Narboni R, 1995, La lumière urbaine, Paris, Le Moniteur

Night Manifesto. Seeking Citizenship 24h, 2014, Sao Paulo, Invisiveis Produçoes

Perrault-Soliveres, A. 2001. Infirmières, le savoir de la nuit, Paris, PUF.

Thoenig J.-C., 2010, "Politique publique", in Boussaguet L. et al., Dictionnaire des politiques publiques, Éditions de Sciences Po, pp. 420-427

Rosa H., 2010, Accélération. Une critique sociale du temps, Paris, La Découverte.

\section{Citer cet article :}

Gwiazdzinski L., 2019, La nuit nouveau territoire des politiques publique, Revue L’Observatoire des politiques publiques, ${ }^{\circ} 53$, hiver 2019, pp.11-15

(*) Luc Gwiazdzinski est géographe, directeur du Master Innovation et territoire à l'université Grenoble Alpes. Il a dirigé une vingtaine de colloques et programmes de recherche sur la nuit et les temps urbains. Il est l'auteur de nombreux articles et ouvrages sur ces questions parmi lesquels : La nuit dernière frontière de la ville (L'aube) ; la Ville $24 \mathrm{~h} / 24$ (L'Aube) ; La Nuit en questions (Hermann) ; Nuits d'Europe (UTBM), L'hybridation des mondes (Elya); Chronotopies (Elya). 